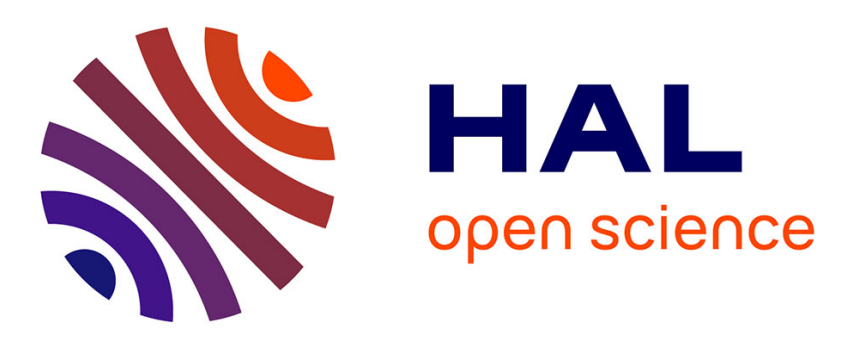

\title{
Glass-Transition Temperature Gradient in Nanocomposites: Evidence from Nuclear Magnetic Resonance and Differential Scanning Calorimetry
}

Aurélie Papon, Hélène Montes, Mohamed Hanafi, François Lequeux, Laurent Guy, Kay Saalwächter

\section{To cite this version:}

Aurélie Papon, Hélène Montes, Mohamed Hanafi, François Lequeux, Laurent Guy, et al.. GlassTransition Temperature Gradient in Nanocomposites: Evidence from Nuclear Magnetic Resonance and Differential Scanning Calorimetry. Physical Review Letters, 2012, 108 (6), pp. 065702. 10.1103/PhysRevLett.108.065702 . hal-01576572

\section{HAL Id: hal-01576572 https://hal.sorbonne-universite.fr/hal-01576572}

Submitted on 23 Aug 2017

HAL is a multi-disciplinary open access archive for the deposit and dissemination of scientific research documents, whether they are published or not. The documents may come from teaching and research institutions in France or abroad, or from public or private research centers.
L'archive ouverte pluridisciplinaire HAL, est destinée au dépôt et à la diffusion de documents scientifiques de niveau recherche, publiés ou non, émanant des établissements d'enseignement et de recherche français ou étrangers, des laboratoires publics ou privés. 


\title{
Glass-Transition Temperature Gradient in Nanocomposites: Evidence from Nuclear Magnetic Resonance and Differential Scanning Calorimetry
}

\author{
Aurélie Papon, ${ }^{*}$ Hélène Montes, Mohamed Hanafi, and François Lequeux \\ PPMD-SIMM, Soft Matter Science and Engineering, UMR 7615 CNRS/UPMC/ESPCI ParisTech, \\ 10 rue Vauquelin, F-75231 Paris Cedex 5, France \\ Laurent Guy \\ Rhodia Opérations, 15 rue Pierre Pays, BP 52, F-69660 Collonges-au-Mont-d'Or, France \\ Kay Saalwächter \\ Institut für Physik-NMR, Martin-Luther-Universität Halle-Wittenberg, Betty-Heimann-Straße 7, D-06120 Halle, Germany
}

(Received 25 May 2011; published 8 February 2012)

\begin{abstract}
The slowing-down of the dynamics of a polymer chain near a surface has been observed for many years now. Here we show that the behavior of model nanocomposites can be quantitatively described with a gradient of glass-transition temperature. We describe with a single parameter-the range of this gradient - the temperature and solvent effect on the spin relaxation dynamics. Moreover, this parameter allows a quantitative description of the nanocomposite calorimetric response from the one of the bulk polymer.
\end{abstract}

DOI: 10.1103/PhysRevLett.108.065702

PACS numbers: 64.70.pj

Dispersing solid fillers into an elastomeric matrix is known to significantly improve the mechanical properties of the material and make them more attractive for industrial applications such as tires. This improvement is not simply the consequence of a geometrical effect, but it is in fact related to a modification of the polymer dynamics in the presence of the fillers. However, the description of this modification is not straightforward, and its interpretation is still debated. Since the 1970s, NMR experiments have shown the existence of different polymer mobility domains in filled elastomers [1,2], and Struik has pointed out, through aging experiments, the presence of a glassy polymer above the glass-transition temperature of the elastomer matrix in those systems [3]. Those observations can be interpreted as the existence of an increase in the glasstransition temperature $T_{g}$ close to the surface of the solid particles, due to attractive interactions between the fillers and the elastomer, even if there is no consensus on this interpretation as of now [4]. In a first approximation, the $T_{g}$ shift can be described with a core-shell image, but with a glassy layer increasing as the temperature decreases towards the glass-transition temperature of the elastomer. This representation gives satisfactory qualitative results at high enough temperature $\left(T>T_{g}+50 \mathrm{~K}\right)$ [5-7]. However, if we look more closely, at lower temperature a core-shell description is not sufficient anymore and a more complex behavior is observed [8]. To get insight in this polymer slowing down, Torkelson and co-workers $[9,10]$ and Forest and co-workers [11] have successfully performed a direct measurement as a function of the distance to the solid surface in thin films. But this direct method is out of reach for nanocomposites.
To overcome this difficulty, we propose here to test an a priori shape of the slowing-down. For that, we use a model of $T_{g}$ gradient near the solid surface. In effect, in thin polymer films, a progressive change in $T_{g}$ has been observed with the film thickness [12-14]. In the case of an attractive interaction between the substrate and the elastomer, an increase in $T_{g}$ is measured [15] and it has been proposed by Long and Lequeux that it could be modeled with a gradient following the equation [16]

$$
T_{g}(z)=T_{g}^{\infty}\left(1+\frac{\delta}{z}\right),
$$

where $z$ is the distance from the solid surface, $T_{g}^{\infty}$ the glasstransition temperature in the bulk, and $\delta$ the characteristic length of the gradient. Using this model and the behavior of the pure elastomer matrix, we will show that we can quantitatively describe the behavior of our model filled rubbers (FRs) in NMR with and without solvent and in differential scanning calorimetry (DSC).

Our model samples are composed of a poly(ethyl acrylate) (PEA) matrix with monodispersed silica particles with a diameter of 28,42 , or $87 \mathrm{~nm}$, grafted with either a coupling agent creating a covalent link between the silica and the polymer [grafter TPM: 3-(trimethoxysilyl)propyl methacrylate] or a covering agent shielding part of the hydrogen bonds between the residual $-\mathrm{OH}$ groups on the silica surface and the polymer [grafter C8TES, or $n$-octyltriethoxysilane] [17]. For PEA, $T_{g}=253 \mathrm{~K}$ at $1 \mathrm{~Hz}$ and $273 \mathrm{~K}$ at NMR frequencies around $10 \mathrm{kHz}$, following the Williams-Landel-Ferry (WLF) law.

We will first show that we can fit the proton NMR relaxation curves of the filled rubbers using simply the 
NMR curves of the pure PEA matrix with the $T_{g}$ gradient model. The fitting method consists in integrating the PEA response around an isolated particle, taking into account the $T_{g}$ gradient:

$$
M^{\mathrm{FR}}(T, t)=\frac{\int_{V_{\mathrm{tot}}} M^{\mathrm{PEA}}\left(T-T_{g}(z), t\right) d V(z)}{V_{\mathrm{tot}}},
$$

where $T_{g}(z)$ follows Eq. (1). The volume of integration corresponds to the mean polymer volume per silica particle: $V_{\text {tot }}=\frac{1-\phi_{\mathrm{Si}}}{\phi_{\mathrm{Si}}} \frac{4}{3} \pi R_{\mathrm{Si}}^{3}$, where $R_{\mathrm{Si}}$ is the silica particle radius and $\phi_{\mathrm{Si}}$ the volume fraction of silica in the sample. Of course this is only a mean value and it is thus not very accurate, but it will not have any major consequence on our calculations since the most important part of the integral is the one close to the silica surface, where the polymer chains are immobilized.

Moreover, we have to take into account the presence of protons in the grafters in our model samples. Since they are covalently attached to the silica surface, the grafters are mainly seen as immobilized, even in the presence of solvent, as it has been shown by NMR in Ref. [8]. We will thus consider a $T_{g}$ gradient starting from a distance $e_{0}$ from the solid surface. The origin of $z$ in Eq. (1) has to be interpreted as the distance $R_{\mathrm{Si}}+e_{0}$ from the center of the particle.

To apply our fitting method we then need a description of the NMR curves of the pure PEA matrix at various temperatures. We have previously shown that the NMR relaxation of the PEA matrix-measured with a magic sandwich echo sequence (MSE) that refocuses the free induction decay-can be fitted with the Andersen-Weiss equation [8]

$$
M^{\mathrm{PEA}}(T, t)=\exp \left[-M_{2} \tau_{c}(T)^{2}\left(e^{-t / \tau_{c}(T)}+\frac{t}{\tau_{c}(T)}-1\right)\right] .
$$

This is a classical empirical equation used to analytically describe NMR signals. The parameter $M_{2}$ is found here to be $5.5 \times 10^{9} \mathrm{~s}^{-2}$, and $\tau_{c}$ depends on the temperature and can be fitted according to the Vogel-Fulcher relation

$$
\tau_{c}(T)=\tau_{0} \exp \left(\frac{A}{T-T_{0}}\right)
$$

where $A=1260 \mathrm{~K}, \tau_{0}=3.10^{-4} \mu \mathrm{s}$, and $T_{0}=156 \mathrm{~K}$. Thus, we know how to analytically describe the PEA relaxation at any temperature between $T_{g}-20 \mathrm{~K}$ and $T_{g}+120 \mathrm{~K}$. In the following, we will consider the fits until the time $t=0.04 \mathrm{~ms}$, which is an appropriate time span to include in an equal manner the glassy response with a characteristic time of $0.02 \mathrm{~ms}$ and the elastomer response.

The fitting method consists in integrating the PEA response around a particle:

$$
\begin{aligned}
M^{\mathrm{FR}}(T, t)= & \frac{V_{\mathrm{imm}} M^{\mathrm{PEA}}\left(T-T_{g}^{\max }(z), t\right)}{V_{\mathrm{tot}}} \\
& +\frac{\int_{V_{g}} M^{\mathrm{PEA}}\left(T-T_{g}(z), t\right) d V(z)}{V_{\mathrm{tot}}},
\end{aligned}
$$

where $V_{\mathrm{imm}} \simeq 4 \pi R_{\mathrm{Si}}^{2} e_{0}$ corresponds to the immobilized protons associated to the grafters, $d V(z)=4 \pi\left(R_{\mathrm{Si}}+e_{0}+\right.$ $z)^{2} d z, V_{g}=V_{\text {tot }}-V_{\text {imm }}$, and $T_{g}(z)$ is described by Eq. (1). In practice, we use a cutoff in $T_{g}(z)$ at $T_{g}^{\max }=T+20 \mathrm{~K}$ to avoid the divergence, without noticeable sensitivity on the computed signal (see Fig. 1). The first term of this equation describes the response of the immobilized molecules at the surface of the filler, that includes the protons belonging to the grafters. We describe their dynamics by the one of the matrix $20 \mathrm{~K}$ below its $T_{g}$, which is enough to account for their immobility in the experimental range of time [8].

First, we fit at the same time the whole set of experimental NMR measurements at various temperatures between 283 and $393 \mathrm{~K}$. The standard deviation is calculated for each parameter pair $\left(e_{0}, \delta\right)$ and a minimum is clearly found [see inset of Fig. 2(a)]. We can see in Fig. 2(a) that the fitting curves corresponding to this parameter pair describe very well the experimental measurements. This has been done on various samples, with different silica amounts and sizes and different grafters with similar results. Thus, with only two parameters, we can fit five curves corresponding to behaviors at different temperatures. For the first time it shows explicitly that a $T_{g}$ gradient can phenomenologically describe very well the polymer dynamics in filled elastomers. The typical value we found for $e_{0}$ is $2 \mathrm{~nm}$ in the case of the grafter TPM, which is in
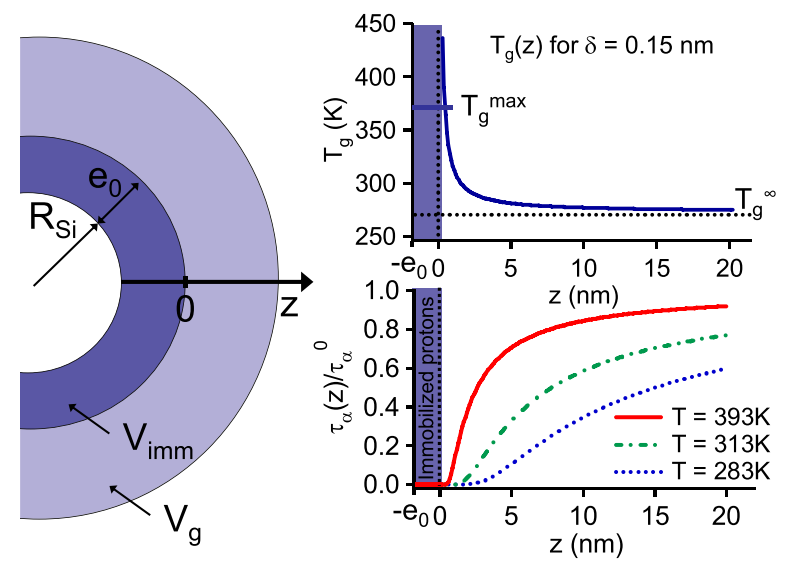

FIG. 1 (color online). Top: Schematic representation of the glass-transition temperature gradient in the vicinity of a particle, with a cutoff at $T_{g}^{\max }$. The volume $V_{\text {imm }}$ represents the immobilized protons at the surface of the particle, corresponding to a thickness $e_{0}, V_{g}$ the volume on which the $T_{g}$ gradient is applied. Bottom: Variation of the ratio of the WLF time at a distance $z$ over the WLF time in the bulk $\tau_{\alpha}(z) / \tau_{\alpha}^{0}$ (the WLF coefficients take into account the $T_{g}$ change at the NMR frequency). It shows the evolution of steepness of the interface with the temperature. 


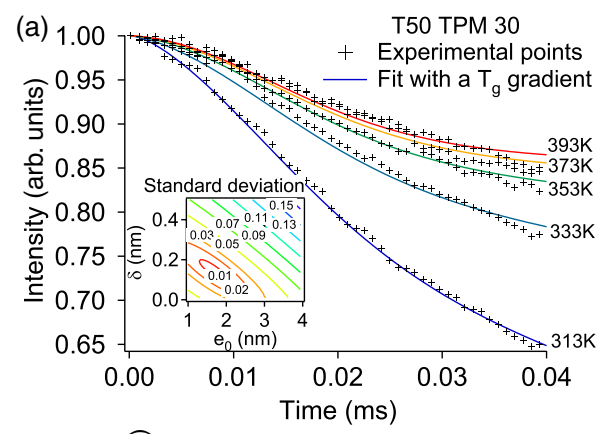

(b)

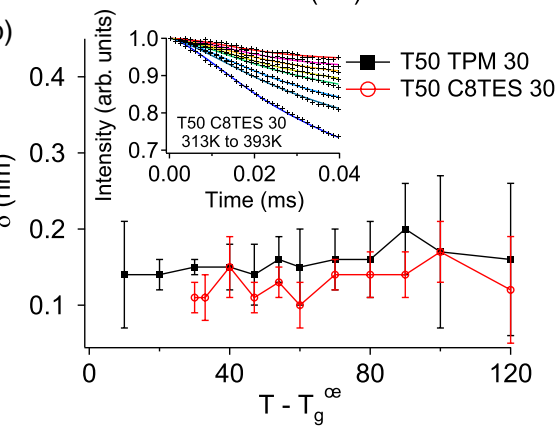

FIG. 2 (color online). MSE refocused free induction decay for filled elastomers fitted with the MSE curves of the PEA matrix taking into account a $T_{g}$ gradient around the fillers. The crosses are used for the experimental signals and the lines for the fits. (a) The fit is done at all the temperatures with the parameters $\left(e_{0}, \delta\right)$ minimizing the standard deviation with the experimental points (inset). For the sample considered here, $R_{\mathrm{Si}}=21.5 \mathrm{~nm}$ and $\phi_{\mathrm{Si}}=0.216$. In the inset, the standard deviation calculated for each parameter pair shows a minimum. (b) In the inset, the fit is done independently at each temperature, with $e_{0}$ fixed from the previous fit. $\delta$ is thus the only free parameter and it is determined for each temperature. For the sample considered here, $R_{\mathrm{Si}}=21 \mathrm{~nm}$ and $\phi_{\mathrm{Si}}=0.179$. We see in the main figure that $\delta$ does not depend on the temperature in this temperature range, neither with the TPM nor with the C8TES grafters.

agreement with the expected grafter thickness. In the case of the grafter C8TES, the value obtained is smaller, around $0.5 \mathrm{~nm}$, because this grafter is expected to be more mobile since it is not covalently linked to the polymer. This is in agreement with the previous results from Ref. [8]. Regarding the parameter of the gradient $\delta$, we found it to be between 0.1 and $0.2 \mathrm{~nm}$ for all the samples, which is of the order of magnitude that we had previously estimated with a core-shell approximation [18]. It is also similar to the values obtained in previous NMR studies in confined systems [19]. Moreover, if we take as "glassy" the polymer below its $T_{g}+20 \mathrm{~K}$, we obtain glassy thicknesses ranging from 1 to $3 \mathrm{~nm}$ for C8TES samples and from 2 to $6 \mathrm{~nm}$ for the TPM samples in the considered range of temperature, which is also in agreement with literature results $[6,20,21]$.

In order to test more accurately the value of $\delta$, we take $e_{0}$ as deduced from the previous fit and examine whether or not $\delta$ depends on the temperature. Whereas the previous fit was done for all the temperatures at the same time, here we fit the NMR curves independently at each temperature from $T_{g}+10 \mathrm{~K}$ to $T_{g}+120 \mathrm{~K}$, with the parameter $\delta$ free. In Fig. 2(b) we show that no significant change in $\delta$ is found, neither with the TPM grafter nor with the C8TES. The coefficient $\delta$, characteristic of the interaction between the filler surface and the polymer, is thus independent of the temperature between $T_{g}+10 \mathrm{~K}$ and $T_{g}+120 \mathrm{~K}$. This proves clearly that the polymer dynamics can be described by a gradient of glass-transition temperature, even at temperatures very close from $T_{g}$ in the bulk.

Moreover, this description is still suitable in the case of the addition of solvent, which is known to decrease the $T_{g}$ of the bulk elastomer.

We considered NMR relaxation curves of the swollen filled rubbers and fitted them with the parameter pair $\left(e_{0}, \delta\right)$ that has been obtained in the previous dry case, keeping only one free parameter, the glass-transition temperature in the bulk $T_{g}^{\infty}$, which is modified in the presence of solvent. Figure 3 shows that the $T_{g}$ shifts deduced from the $T_{g}$ gradient model and the ones measured in DSC on the pure matrix in the presence of solvent are very well correlated. This is another evidence that a $T_{g}$ gradient model describes well the polymer dynamics in our model filled rubbers.

Finally, another classical signature of the glass transition in elastomers is their DSC response. We have thus examined the validity of our model with this technique.

First, in Fig. 4(a) we compare the $T_{g}$ measured on a filled sample with the prediction from the $T_{g}$ gradient model. The results are obtained at a cooling or heating rate of $10 \mathrm{~K} \mathrm{~min}^{-1}$. In a similar manner as previously, we integrated the heat capacity of the PEA matrix $c_{p}^{\mathrm{PEA}}\left(T-T_{g}(z)\right)$ around one silica particle, taking into account the gradient

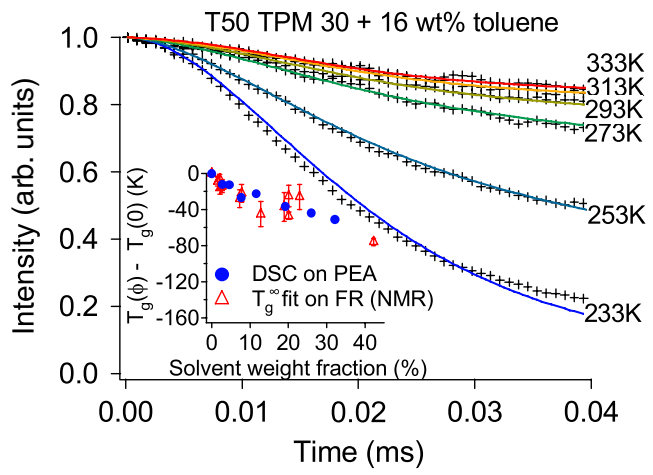

FIG. 3 (color online). The MSE refocused free induction decay of filled samples with solvent can be fitted with the $T_{g}$ gradient model with only one free parameter: $T_{g}^{\infty}$, which is the $T_{g}$ in the bulk PEA with solvent. The parameter pair $\left(e_{0}, \delta\right)$ is not expected to change with the addition of solvent and is thus fixed at its value in the dry case. The sample used is the same as in Fig. 2(a). In the inset, comparison between $T_{g}$ shifts measured in NMR and in DSC. FR denotes filled rubbers. A good agreement is observed. 
(a)

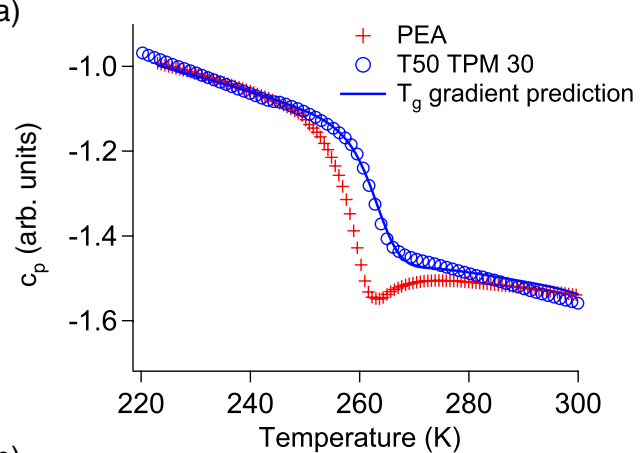

(b)

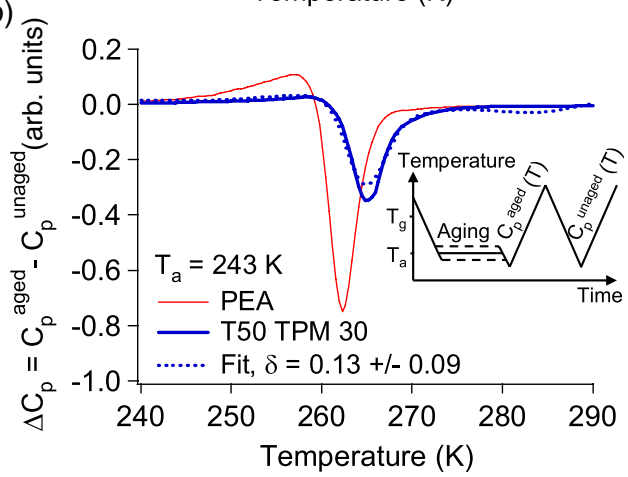

FIG. 4 (color online). (a) Heat capacity measurement for the pure PEA matrix (crosses) and a filled sample (open circles). The sample is the same as in Fig. 2(a). The line is the prediction for the filled sample made from the behavior of the pure matrix and a $T_{g}$ gradient. (b) In the inset, thermal profile used for the aging experiments. The cooling and heating rates are all $10 \mathrm{~K} \mathrm{~min}^{-1}$. The figure presents $\Delta c_{p}\left(T_{a}, T\right)$ measured for the PEA matrix (thin line) and the same filled sample as in (a) (thick line) aged at $253 \mathrm{~K}$. The fit obtained with the $T_{g}$ gradient model is plotted with a dashed line. The parameter $e_{0}$ was fixed at the value obtained from the NMR fit and $\delta$ was free. The values found for $\delta$ are very close to the ones obtained from the NMR fit.

$T_{g}(z)$ with the parameters determined previously from the NMR relaxation curve. There is thus no adjustable parameter, and we can see that the prediction is in very good agreement with the experimental DSC measurement, both in the amplitude of the transition and in the shift of $T_{g}$.

In the end, we show the robustness of the model with the fit of the heat capacity of filled rubber after aging with measurements done on the pure elastomer matrix. For that, we measured $c_{p}^{\mathrm{PEA}}\left(T_{a}, T\right)$ for the PEA matrix after aging $10 \mathrm{hr}$ at various temperatures $T_{a}$ between 223 and $253 \mathrm{~K}$ [see Fig. 4(b)]. For convenience, we worked on the difference $\Delta c_{p}\left(T_{a}, T\right)=c_{p}^{\text {aged }}\left(T_{a}, T\right)-c_{p}^{\text {unaged }}(T)$. As previously, we fit the behavior of a filled sample by integration around a filler

$$
\Delta c_{p}^{\mathrm{FR}}\left(T_{a}, T\right)=\frac{\int_{V_{g}} \Delta c_{p}^{\mathrm{PEA}}\left(T_{a}-T_{g}(z), T-T_{g}(z)\right) d V(z)}{V_{g}} .
$$

Here, we fix the parameter $e_{0}$ to the value found in NMR and we let $\delta$ free. We see in Fig. 4(b) that the fit from the $T_{g}$ gradient model is rather close to the experimental measurement done in DSC, with a parameter $\delta$ very close to the one found in NMR $(\sim 0.15 \mathrm{~nm})$. If the temperature of the peak is very well described, the amplitude of the peak is more difficult to reproduce since it also depends on the quality of the contact of the sample with the heating plate during the DSC measurement, which is hardly reproducible from one sample to another. The agreement between the experimental curve and the prediction confirms nevertheless that this model of $T_{g}$ gradient around the particles is able to describe the polymer relaxation seen through NMR experiment or through DSC experiment with the same parameters.

To sum up, the physical picture that follows this $T_{g}$ gradient model in nanocomposites is schemed in Fig. 1. It shows a layer of glassy polymer around the fillers that is connected to the elastomer network through an interface whose steepness depends on the temperature. The closer the temperature to the bulk $T_{g}$, the larger the glassy layer and the more gradual the interface.

We saw through different experiments that the behavior of our model filled elastomers can be very well described using the behavior of the pure elastomer matrix and a $T_{g}$ gradient in the vicinity of the fillers. This is the first direct interpretation of experimental data using explicitly a gradient, and the strength of the model has been shown through its ability to describe both NMR measurements with and without solvent and DSC measurements.

In conclusion, the amplitude of the slowing-down of the polymer in our model nanocomposites can be interpreted as a distribution of glass-transition temperatures following the $T_{g}^{\infty}(1+\delta / z)$ equation.

We gratefully acknowledge Roberto Perez Aparicio and Paul Sotta for their help with the NMR measurements made at the LPMA (CNRS/Rhodia) in Lyon. This work has been supported by the ANR grants Dynafil and TaylRub.

*Present address: Rhodia Opérations, 15 rue Pierre Pays, BP 52, F-69660 Collonges-au-Mont-d’Or, France. aurelie.papon@espci.org "www.ppmd.espci.fr/?lang=en

[1] S. Kaufman, W. Slichter, and D. Davis, J. Polym. Sci., A2, Polym. Phys. 9, 829 (1971).

[2] V. Litvinov and P. Steeman, Macromolecules 32, 8476 (1999).

[3] L. Struik, Polymer 28, 1521 (1987).

[4] G. McKenna, Eur. Phys. J. Special Topics 189, 285 (2010).

[5] J. Berriot, F. Lequeux, L. Monnerie, H. Montes, D. Long, and P. Sotta, J. Non-Cryst. Solids 307-310, 719 (2002). 
[6] J. Berriot, H. Montes, F. Lequeux, D. Long, and P. Sotta, Macromolecules 35, 9756 (2002).

[7] J. Berriot, H. Montes, F. Lequeux, D. Long, and P. Sotta, Europhys. Lett. 64, 50 (2003).

[8] A. Papon, K. Saalwächter, K. Schäler, L. Guy, F. Lequeux, and H. Montes, Macromolecules 44, 913 (2011).

[9] C. Ellison and J. Torkelson, Nature Mater. 2, 695 (2003).

[10] S. Kim, S. A. Hewlett, C. B. Roth, and J. M. Torkelson, Eur. Phys. J. E 30, 83 (2009).

[11] J. Mattsson, J. A. Forrest, and L. Borjesson, Phys. Rev. E 62, 5187 (2000).

[12] J. Keddie and R. Jones, Isr. J. Chem. 35, 21 (1995).

[13] D. Fryer, R. Peters, E. Kim, J. Tomaszewski, J. de Pablo, P. Nealey, C. White, and W. Wu, Macromolecules 34, 5627 (2001).
[14] C. Rotella, S. Napolitano, L. D. Cremer, G. Koeckelberghs, and M. Wübbenhorst, Macromolecules 43, 8686 (2010).

[15] D. Fryer, P. Nealey, and J. de Pablo, Macromolecules 33, 6439 (2000).

[16] D. Long and F. Lequeux, Eur. Phys. J. E 4, 371 (2001).

[17] J. Berriot, H. Montes, F. Martin, M. Mauger, W. PyckhoutHintzen, G. Meier, and H. Frielinghaus, Polymer 44, 4909 (2003).

[18] H. Montes, F. Lequeux, and J. Berriot, Macromolecules 36, 8107 (2003).

[19] S. Gradmann, P. Medick, and E. A. Roissler, J. Phys. Chem. B 113, 8443 (2009).

[20] W. Zheng and S. L. Simon, J. Chem. Phys. 127, 194501 (2007).

[21] S. Napolitano, V. Lupascu, and M. Wubbenhorst, Macromolecules 41, 1061 (2008). 